\title{
LS FITTING OF THE MATERIAL PROPERTIES INFLUENCING THE PRINT QUALITY IN FLEXOGRAPHIC REPRODUCTION SYSTEM
}

\author{
Tamara Tomašegović (iD), Tomislav Cigula (iD), Juraj Huzjak (iD), Marija Prša (D) \\ University of Zagreb, Faculty of Graphic Arts, Zagreb, Croatia
}

\begin{abstract}
Printing quality is influenced by numerous parameters and by the material interactions in the inkplate-substrate system. The aim of this paper is to characterize and quantify influential parameters in the flexographic reproduction system by using the method of the least squares (LS fitting). Specifically, this paper deals with influential properties of the printing plates and porous printing substrates (paper, board). Printing plate with its properties and interaction with the printing ink and substrate during the printing process affects the parameters of the print. Different properties of analysed porous substrates define properties of the print such as tonal values/dot gain and optical density. Measured properties of different printing substrates (papers and boards with different types of coatings) are used to build a quantification system of influencing parameters for the quality of the print using two different types of printing plates (patterned and non-patterned). Substrate parameters included in this research are roughness, surface free energy and gloss, while the surface free energy analysis was used to define printing plate properties. Results have shown that the least squares method can be applied to define the suitability of a particular type of printing plate for printing on different materials and enable optimization when selecting the material parameters in the flexographic reproduction system.
\end{abstract}

Key words: flexography, printing plate, printing substrate, paper, board, LS fitting

\section{INTRODUCTION}

Flexography is a printing technique used primarily in the packaging and label production, as well as in novel applications such as functional printing. It utilizes an elastic photopolymer printing plate, which enables the usage of different types of substrates (Kipphan, 2001; Page Crouch, 1998).

Many parameters influence the quality of the print in flexography: photopolymer plate used for the printing plate production (Matsubara et al, 2011), bump and compensation curves applied to the plate, properties of anilox roller, type of tape placed under the printing plate to adjust its deformation, properties of the printing ink and printing substrate (Bollström et al, 2013) and control of the printing process (Mahović Poljaček et al, 2013).

Improvements of the flexographic printing plate's mechanical and surface properties in the past decade have been implemented in order to widen the possibilities of the application of flexography in graphic reproduction. For example, patterned textures have been applied to the surface of the printing elements on the printing plate in the plate making process (Kodak, n.d.); (Flint, n.d.). The increased surface roughness of the printing plate consequently enables better adsorption of the printing ink to the printing plate, and improved transfer of the ink to the printing substrate, reducing the fingering and enhancing the visual impression of the print. Surface patterns can be applied to the plate only in high coverage area $(>80 \%)$ or in the whole range of tonal values, depending on the type of the ink, substrate and printing plate itself.

Different types of printing substrates can be used in flexography. Among them, foil and paper/board are the most common. Adjustment of the printing plate's coverage values is necessary for each printing substrate because of the influence of the substrate's properties on the transfer and behaviour of the ink on its surface in the printing process.

Main properties of the printing substrate that influence the properties of the print to extent are roughness, surface free energy, smoothness and gloss. Each of these properties in relation to the properties of the printing plate itself will result with different quality of the print properties (optical density and tonal value, i.e. tone value increase (Johnson, 2008; Tomašegović, 2016.).

The aim of this paper was to quantify the influence of the parameters of the printing substrate on the properties of the print, when using patterned and non-patterned printing plate. In order to achieve this, least square (LS) fitting in matrix form was applied. LS fitting method enabled assigning the "weight coefficients" to each of the substrate parameters that affect the properties of the print (optical density and tonal values). In this way, the strength of the each property of printing substrate related to optical density and specific tonal value $(5 \%, 40 \%, 80 \%)$ on the print could be assessed. 
The quantification of the substrate parameters influencing the print quality in flexography can be used to predict and assess the suitability of a particular printing substrate for the usage with the chosen flexographic printing plate and ink.

\section{MATERIALS AND METHODS}

For this research, four types of paper/board printing substrates were used: matt $100 \mathrm{~g} / \mathrm{m}^{2}$, offset gloss $115 \mathrm{~g} / \mathrm{m}^{2}$, coated $245 \mathrm{~g} / \mathrm{m}^{2}$ and silk $350 \mathrm{~g} / \mathrm{m}^{2}$. Properties of substrates, including roughness, gloss and surface free energy were determined prior to printing.

Measurements of the substrate roughness were performed with a TR200 roughness tester (PortableTesters.com, n.d.). Roughness parameters were determined by measuring ten measurements on the printing side of the substrate. Five measurements were performed in the direction of paper grain and five measurements perpendicular to the paper grain direction.

For the purposes of this experiment, the value taken into account was Ra parameter (the arithmetic mean of the profile in the individual measurement).

Paper gloss was measured using a gloss meter Elcometer 407, which measures reflective glare. Light intensity is registered over a small range of reflected angles. The intensity of the light depends on the material and the angle of illumination. For the purpose of obtaining correct results of the measurements, ranging from high gloss to matt surfaces, three different geometries and three different ranges (BYK, n.d.) have been defined. All surfaces were measured at an angle of $20^{\circ}, 60^{\circ}$ and $85^{\circ}$.

In order to calculate the surface free energy of the printing substrates, contact angles of three probe liquids (water, glycerol, diiodomethane) were measured on each substrate by means of goniometer Dataphysics OCA 30. Temperature set for the measurements was $23 \pm 1{ }^{\circ} \mathrm{C}$. Eight drops of $1 \mu \mathrm{l}$ were applied on the solid surface (printing substrate), and the contact angle was measured 4 seconds after the drop has touched the solid surface. Sessile drop method was used for the measurements. After that, surface free energy (SFE) and its polar $\left(\mathrm{SFE}^{\mathrm{p}}\right)$ and dispersive $\left(\mathrm{SFE}^{\mathrm{d}}\right)$ components were calculated using OWRK method (Owens et al, 1969.).

Materials used for the experiment were commercial styrene-isoprene-styrene based LAMS photopolymer flexographic printing plates and black UV flexographic ink. Two variations of printing plates made from the same material were used: patterned and non-patterned type. The purpose of the surface pattern on the flexographic printing plates is to increase the surface roughness and consequently the amount of the ink transferred to the printing substrate. Pattern was applied to the surface of the printing plate in the whole coverage area: from $1 \%$ to $100 \%$. Surface free energy of printing plates was $~ 30 \mathrm{mN} / \mathrm{m}$, of which up to $0.5 \mathrm{mN} / \mathrm{m}$ consisted of polar component, and the rest of the dispersive.

Printing process was carried out by means of IGT F1 unit (IGT, n.d.). Prior to printing on the substrates used in this experiment, both types of plates were printed with using 10 probe samples in order to stabilize the printing process. Since different types of printing substrates were used, no compensation curves were applied during the production of the plates. Printing parameters, which were kept fixed during the printing process, were adjusted by aiming the optical density of 1.5 on 100 matt paper. Anilox force was set to $300 \mathrm{~N}$; printing force to $50 \mathrm{~N}$, and printing speed was $0.3 \mathrm{~m} / \mathrm{s}$.

After the printing process, optical density (D) and tonal values (TV) on the prints were measured by means of $X$-rite eXact device. Tonal value was measured on $5 \%, 40 \%$ and $80 \%$ of nominal coverage value in order to examine highlights and mid-tones, as well as shadows.

Collected results were used to build a matrix for least square (LS) fitting (Björck, 1996.). Input parameters were Ra, gloss, SFE ${ }^{p}$ and SFE ${ }^{d}$ of the substrates. The reason for not using the total SFE of substrates alongside its components was the singularity of the constructed matrix, which would compromise the accuracy of the results. Output matrixes consisted of vectors representing D and TV at 5\%, 40\% and $80 \%$ for patterned and for the non-patterned printing plates. All input parameters were normalized (0-1). In this way, eight LS fitting sets were constructed. The weight coefficients for each of the substrate parameters (Ra, gloss, SFE ${ }^{p}$ and SFE ${ }^{d}$ ) influencing the print properties (D, TV) were estimated in this way. The results were compared for the prints obtained with patterned and non-patterned printing plate in order to assess the qualitative variations of the print when using different substrate-plate combinations (Johnson, 2008.). 


\section{RESULTS AND DISCUSSION}

\subsection{Properties of printing substrates}

In order to quantify the influence of the chosen parameters of the printing substrate that influence the properties of the print, the variation of their features needed to be present. Figure 1 displays the measured properties of chosen printing substrates.

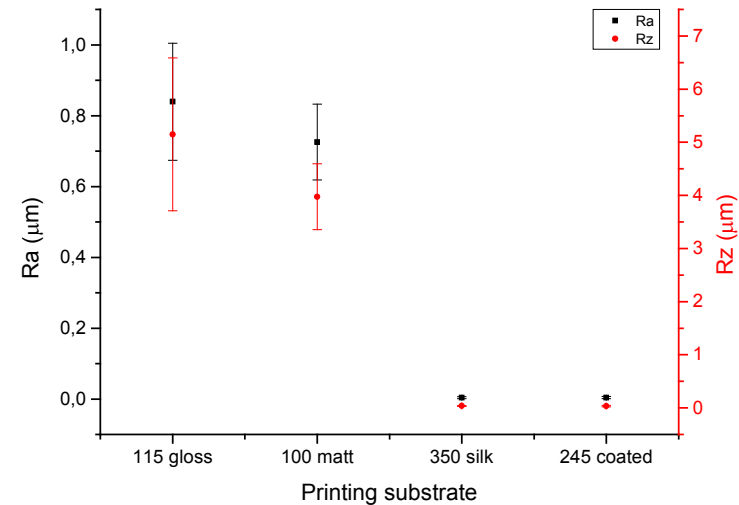

a)

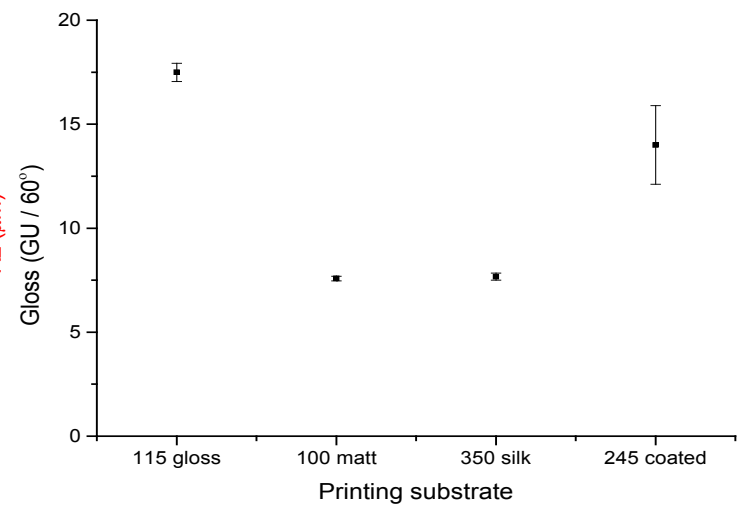

b)

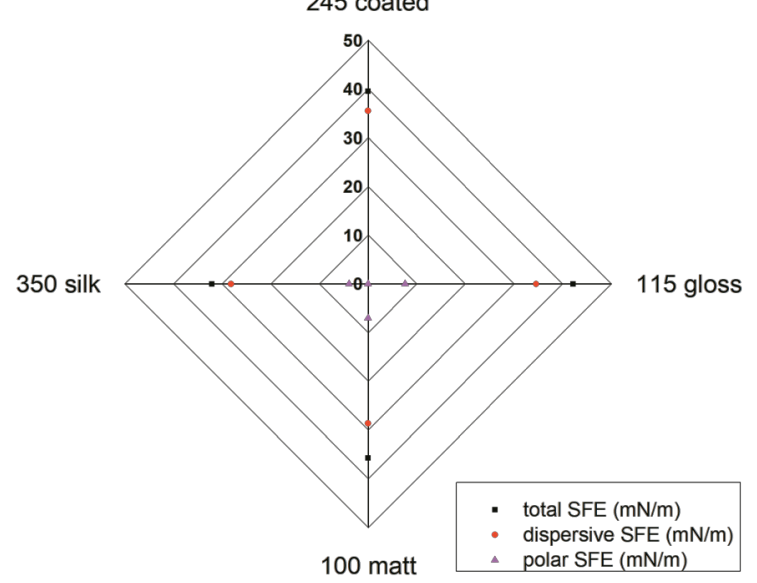

c)

Figure 1. Measured properties of printing substrates: a) roughness parameter $R a, b)$ gloss, c) surface free energy

For the inclusion in matrix prepared for LS fitting, only Ra parameter (Figure 1a) was chosen to represent roughness in the model, since $\mathrm{Ra}$ and $\mathrm{Rz}$ are not independent. Furthermore, Ra parameter showed less deviation. The range of measured Ra was from $0.004 \mu \mathrm{m}$ to $0.84 \mu \mathrm{m}$, depending on the printing substrate.

Values of gloss measurements chosen for the LS fitting were fixed at $60^{\circ}$ in order for them to be comparable and assure the validity of the model. In Figure $1 \mathrm{~b}$, one can see that the values of the gloss for measured surfaces range from $7.5-18 \mathrm{GU}$. Highest deviations of the measurements are present for the 245 coated substrate.

Figure 1c presents the surface free energy (SFE) components of the printing substrates used in this research. Substrate 115 gloss has the highest value of the total SFE at $42.15 \mathrm{mN} / \mathrm{m}$. 350 silk presents the lowest value of total SFE: $32.1 \mathrm{mN} / \mathrm{m}$. All substrates have a dominant dispersive SFE component, with polar component lower than $10 \mathrm{mN} / \mathrm{m}$. Specifically, lowest polar component is present for 245 coated substrate, and it amounts only $0.01 \mathrm{mN} / \mathrm{m}$.

\subsection{Properties of prints}

Figure $2(a-c)$ presents the results of measurements performed on the prints obtained by patterned and non-patterned printing plate. Results of the tonal value measurements are presented in Figure $2 a-b$, and optical densities on the prints are visible in Figure 2c. 
Measured tonal values show the similar trend for the prints obtained by patterned and non-patterned printing plate. Substrates 350 silk and 245 coated present lower tonal values than substrates 100 matt and 115 gloss. This can be explained by the higher roughness of substrates 100 matt and 115 gloss (Figure 1a).

When comparing the tonal values obtained by patterned and non-patterned printing plate on the same substrate, it is visible that the patterned printing plate produces higher tonal value increase - because of the higher amount of the ink present on the plate itself and therefore transferred to the print.

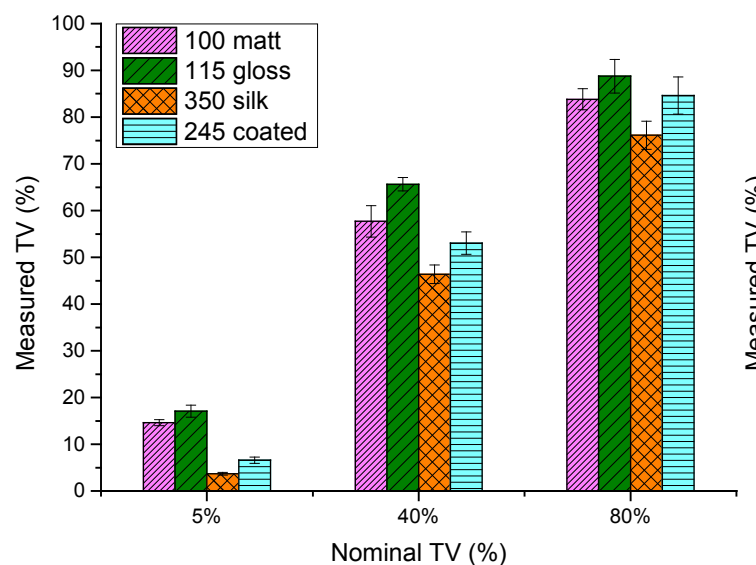

Nominal TV $(\%)$

a)

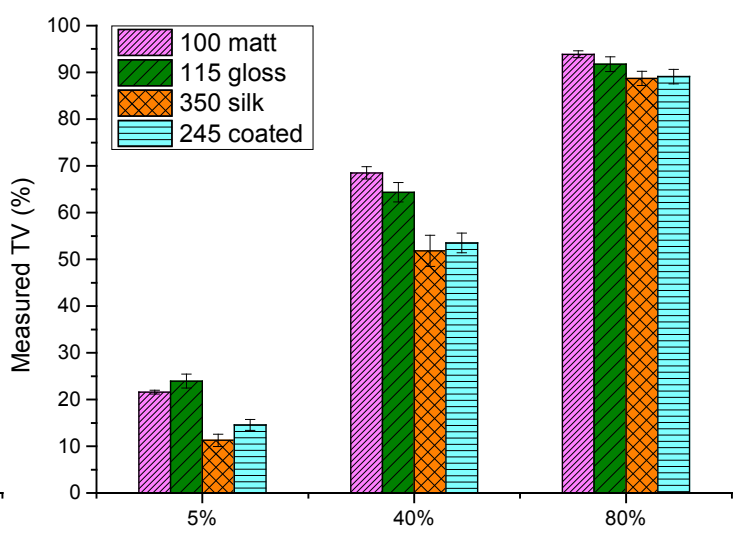

Nominal TV $(\%)$

b)

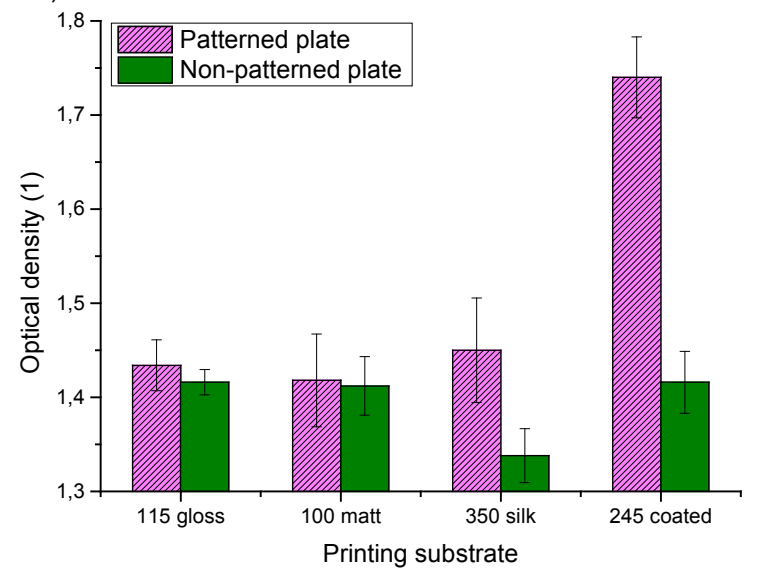

c)

Figure 2. Measured properties of prints: a) tonal values obtained by non-patterned printing plate, b) tonal values obtained by patterned printing plate, c) optical density

Optical density measured on the prints is highest when using the patterned printing plate and 245 coated substrate; and lowest when using non-patterned plate and 350 silk substrate (Figure 2c). As expected, optical density on the substrate is higher when printing with the patterned printing plate, specifically on the substrate that possess low roughness ( 350 silk and 245 coated).

Furthermore, the print on substrate 245 coated with the patterned printing plate has significantly higher optical density than the rest of the printed samples. This could be explained by the almost non-existent polar component of this substrate - specifically, of the substrate's coating. When transferring a higher amount of the ink with the patterned printing plate to this substrate, due to the properties of the coating, penetration of the ink into the substrate before the drying could be decreased. This will result with higher measured optical density.

It can be concluded that all presented printing substrate parameters have an impact on the tonal values and optical density on the prints obtained by patterned and non-patterned printing plates. However, the effects of those parameters are individual and different for each substrate property.

It is therefore necessary to quantify those influences in order to assess their degree of impact on the properties of the flexographic print. 


\subsection{LS fitting}

After analysis of the results, the application of the least squares method in the matrix form (LS fitting) was applied in order to interpret the obtained measured values in the context of the quality of the prints. The aim was to predict the trends of optical density and tonal values on prints produced using different printing substrates and patterned/non-patterned printing plates. Figure 3 presents the weight coefficients for chosen parameters of the substrates that influence the properties of the print when using patterned and non-patterned plate. The impact can have a positive or negative sign, presenting the proportional or inversely proportional connection, respectively.

It is visible that some parameters have generally negligible effect on the properties of the print, specifically polar component of the surface free energy of the substrate (with the exception of the influence on optical density when using rough printing plate, Figure $3 a-c)$, due to its low values on substrates and printing plates compared to the dispersive component. The obtained results point to the dominant impact of the roughness of the substrate, gloss and dispersive component of the surface free energy on the analysed properties of the print.

The influence of the substrate roughness with positive sign is present for both printing plates for the tonal values on the prints (Figure 3a-b). This influence was similar for all tonal areas: highlights, mid-tones and shadows. Higher roughness of the substrate will generally, regardless of the type of printing plate, result with a higher tone value increase on the print due to the deformation of the screen element on the print.

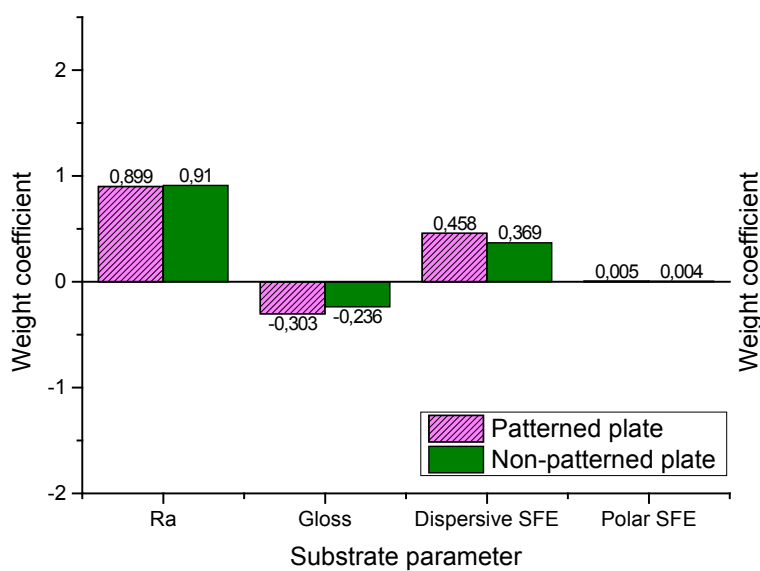

a)

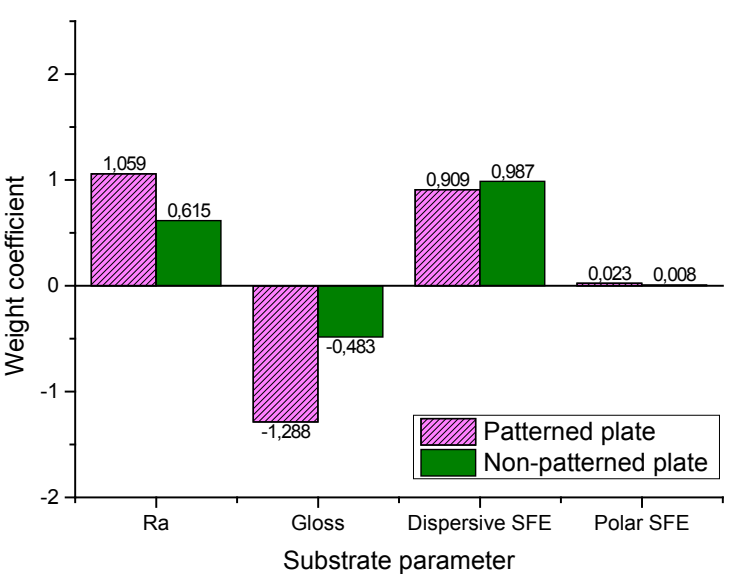

b)

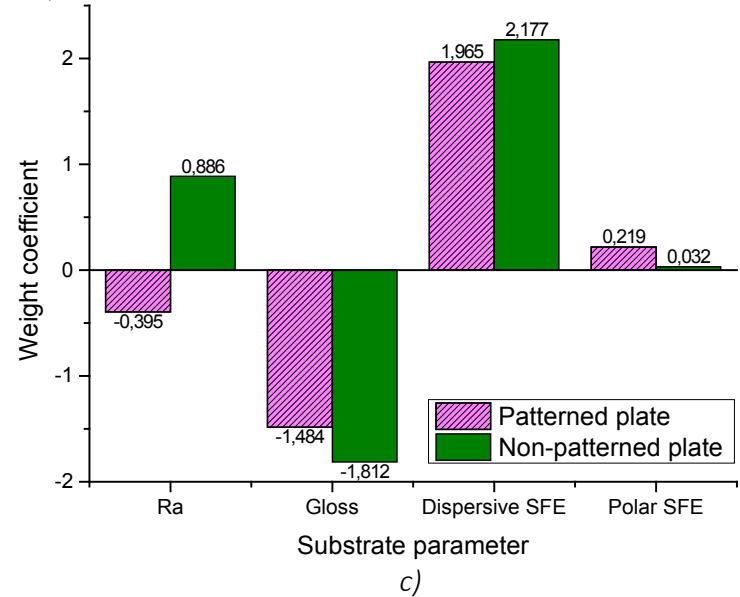

Figure 3. Weight coefficients of influential parameters of substrates for: a) tonal value of $5 \%$ on prints, b) tonal value of $80 \%$ on prints, c) optical density on prints

The roughness of the substrate affects positively the optical density on the prints obtained by nonpatterned printing plate, while the influence on the prints obtained by patterned printing plate is negative (Figure 3c). It is visible that the transfer of the printing ink (and thus the optical density on the print) is improved when a rough and smooth surface (rough printing plate and smooth printing substrate 
or vice versa) are used in the printing process. In the smooth-to-smooth or rough-to-rough system, amount of the transferred ink will be decreased.

Other substrate parameters (gloss, dispersive and polar surface free energy) influence the tonal values with the same sign throughout the measured range, regardless of the type of used printing plate.

Gloss, which can be associated with the type of the substrate coating, has an impact with a negative sign on the optical densities and increase of the tonal values. It can be concluded that the increased gloss of the substrate due to the use of specific coatings reduces the ability of the ink adsorption on the printing substrate and its penetration into the substrate. Therefore, optical density and the increase of tonal values on the print (dot gain) are reduced compared to the prints on substrates with coatings which do not possess, among other properties, high gloss.

The dispersive component of surface free energy has an influence with positive sign. The UV flexographic ink used in this experiment is mainly composed of non-polar components (polymers, oligomers, waxes, etc.). Therefore, when the contact with the printing substrate with pronounced dispersive component of surface free energy is achieved, the ink transfer is improved. This will be manifested by the increased tonal values on the print, as well as with increased optical density.

The LS fitting made it possible to connect the properties of the printing substrate and the print when varying the media between them in the printing process - printing plate. The results obtained by the LS method provided a mathematical weight to the interpretations of the influence of printing substrates and plates on flexographic print, specifically in the case of evaluation and linking of multiple parameters.

\section{CONCLUSIONS}

The aim of this research was to quantify the influential parameters in the flexographic reproduction system using the least squares method, and to define the interaction of a particular type of printing substrate and printing plate. This enables the optimization of the flexographic printing system.

Roughness, gloss and surface free energy were measured on the chosen printing substrates, and the printing process was carried out using patterned and non-patterned printing plate. Obtained prints were used to measure optical density (D) and tonal values (TV) on prints at certain percentages (5\%, 40\% and $80 \%)$. Based on the collected data, a model presenting the weight coefficients of the influential parameters in flexographic system was developed using the least squares method. Fitting was applied for both patterned and non-patterned printing plate.

Based on the obtained results, one can conclude that optical density is enhanced when a combination of smooth printing substrate and rough printing plate (or vice versa) is used in the reproduction process. Furthermore, increased roughness of the printing substrate will result with increased tonal values on the print. Increased gloss has an impact with negative sign on optical density and dot gain on prints produced by both types of printing plates due to the surface properties of the coatings and decreased penetration of the ink in the coated paper/board.

Increased dispersive component of the surface free energy has a positive effect on the increase of tonal values and optical density on the print when using chosen standard UV flexographic ink.

Polar component of surface free energy, due to its low values on both printing plate and substrate, has a weak impact on the properties of the print.

This research has shown that the qualitative properties of the prints (represented by D and TV) obtained by the materials used in this experiment are dominantly affected by roughness, gloss and dispersive component of the surface free energy of the substrate. Furthermore, it is possible to quantify the influence of these parameters using LS fitting.

In accordance with the qualitative requirements, it is therefore possible to choose a combination of patterned/non-patterned printing plate and substrate in order to optimize the flexographic printing system.

\section{ACKNOWLEDGMENTS}

This research is part of the project UIP-2017-05-4081, Development of the model for production efficiency increase and functionality of packaging, supported by Croatian Science Foundation. 


\section{REFERENCES}

[1] Björck, A.: "Numerical methods for least squares problems", PhD thesis, Linköping University, Linköping, 1996.

[2] Bollström, R., Tobjörk, D., Dolietis, P.,Salminen, P., Preston, J., Österbacka, R., Toivakka, M.: "Printability of functional inks on multilayer curtain coated paper", Chemical Engineering and Processing: Process Intensification 68, 13-20, 2013. doi: 10.1016/j.cep.2012.07.007.

[3] BYK, Gloss measurement, BYK-Gardner GmbH, URL:

[4] https://www.byk.com/fileadmin/byk/support/instruments/theory/appearance/en/Intro_Gloss.pdf (last request: 2018-09-16).

[5] Flint, Flint NeXT technology, Flint Group, URL: https://www.flintgrp.com/en/products/flexographic/nyloflex-next/ (last request: 2018-09-16).

[5] IGT, IGT: F1 printability tester, IGT Testing Systems, 2016, URL: http://www.igt.nl/image/data/IGT/Downloads/IGT\%20F1\%20brochure\%20(ENG).pdf (last request: 2018-09-16).

[6] Johnson, J.: "Aspects of flexographic print quality and relationship to some printing parameters", PhD thesis, Karlstad University, 2008.

[7] Kipphan, H.: "Handbook of Print Media", (Springer, Berlin, 2001).

[8] Kodak, Kodak NX technology, Kodak, URL: http://graphics.kodak.com/KodakGCG/uploadedFiles/Products/Computer-toplate/Flexo_CTP/TRENDSETTER_NX_Imager/Tab_Contents/TSNX_sellsheet.pdf (last request: 2018-09-16).

[9] Mahović Poljaček, S., Cigula, T., Tomašegović, T.: "Meeting the quality requirements in flexographic plate making process", Proceedings of IC 2012, (Budapest, Hungary, 2012), pages 62-69.

[10] Matsubara, T., Oda, R.: Pat. 20,110,308,412, "Block copolymer composition for flexographic printing plates", 2011.

[11] Owens, D. K., Wendt, R. C.: "Estimation of the surface free energy of polymers", Journal of Applied Polymer Science 13(8), 1741-1747, 1969. doi: 10.1002/app.1969.070130815.

[12] Page Crouch, J.: "Flexography Primer", $2^{\text {nd }}$ ed, (PIA/GATF Press, Pittsburgh, 1998).

[13] PortableTesters.com, TR200 Surface Roughness Tester, PortableTesters.com, URL: https://portabletesters.com/product/tr-200-surface-roughness-tester/ (last request: 2018-09-19).

[14] Tomašegović, T.: "Fuctional model of photopolymer printing plate production process", PhD thesis, University of Zagreb, 2016.

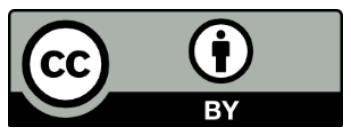

(C) 2018 Authors. Published by the University of Novi Sad, Faculty of Technical Sciences, Department of Graphic Engineering and Design. This article is an open access article distributed under the terms and conditions of the Creative Commons Attribution license 3.0 Serbia (http://creativecommons.org/licenses/by/3.0/rs/). 